\title{
COLLECTIVE EFFECTS IN LEPTON RING OF ERHIC
}

\author{
D. Wang, M. Farkhondeh, J. van der Laan, Ch. Tschalaer, F. Wang, A. Zolfaghari, T. Zwart, \\ Bates Laboratory, Massachusetts Institute of Technology, Middleton, MA 01949, USA \\ M. Blaskiewicz, Y. Luo, L. Wang, Brookhaven National Laboratory, Upton, NY 11973, USA
}

\begin{abstract}
The lepton ring of eRHIC is being designed to provide intense, highly polarized electron and positron beams at 5$10 \mathrm{GeV}$ energy range for the high luminosity leptonhadron collisions. In this paper the estimates of major beam instabilities in the lepton storage ring are presented. Besides conventional impedance-driven instablities the fast beam-ion instability and electron cloud effects are major concerns. The studies suggest that those instbilities would not limit the performance of the lepton ring of eRHIC.
\end{abstract}

\section{INTRODUCTIONS}

The eRHIC is a new lepton-hadron collider undergoing design studies by a collaboration of Brookhaven National Laboratory, MIT-Bates Laboratory, DESY (Hamburg) and BINP (Novosibirsk). The collider complex will consist of a hadron machine that is mainly the existing RHIC (Relativistic Heavy Ion Collider) with necessary upgrades, and a new lepton machine that can provide intense ( $0.5 \mathrm{~A}$ or higher), highly polarized electron and positron beams at energy of $5-10 \mathrm{GeV}$.

Table 1: Nominal parameters of lepton ring of eRHIC

\begin{tabular}{lc}
\hline \hline Energy $(\mathrm{GeV})$ & $5-10$ \\
Circumference (m) & 1278 \\
RF frequency (MHz) & 478.6 or 506.6 \\
RF voltage (MV) & $5-25$ \\
Total current (A) & 0.45 \\
Particle/bunch $\left(10^{11}\right)$ & 1.0 \\
Bunch spacing (m) & 10.6 \\
Momentum comp. & $0.009 / 0.0026$ \\
Energy loss/turn (MeV) & $0.72 / 11.7$ \\
Average beta(m) & $\sim 15$ \\
Bunch length $(\mathrm{cm})$ & $1 \sim 2$ \\
\hline \hline
\end{tabular}

In the current eRHIC design, the bunch spacing is primarily determined by the existing hadron machine complex. There is little flexibility for generating different bunch patterns. One has to deal with a high bunch current and a relatively high total beam current. Since the bunch length (hence the beta-functions at IP) of the hadron beams is longer than $10 \mathrm{~cm}$, the bunch length of lepton beams $(1-2 \mathrm{~cm}$ is expected) is not an issue. The main concern for single bunch effect is the transverse modecoupling instability. We also discuss the power deposition generated by a beam in the form of the higher order mode (HOM) losses by interacting with its surroundings. The narrow-band impedance and related instabilities need to be evaluated carefully due to the relatively large number of RF cavities. The eRHIC machine is planned to operate over a wide range of beam energies. Many collective effects exhibit their strongest behavior at low energy where the beam is less rigid and damping time is much longer than at higher energies. Since electron and positron beams are required by the physics programs, the lepton machine has to account for both electron cloud effects (ECE) for positron operation, and fast beam-ion instability (FBII) for electron operation in the ring design.

In terms of collective effects, several issues are of particular concern including:

- Single bunch instabilities

- Higher-order-mode (HOM) heating

- Coupled bunch instabilities

- Ion related effects

- Electron cloud effects, etc.

\section{IMPEDANCE-RELATED INSTABILITIES}

A preliminary impedance budget is made based on the current machine design. One of the major sources of impedance is RF cavities. In this work the SLAC copper cavity and KEK superconducting cavity that proved very successful in high current operations in B-Factories are used in the estimates of impedance and instabilities.

Table 2: Impedance and loss factor budgets

\begin{tabular}{|c|c|c|}
\hline component & $\begin{array}{c}\text { Inductive } \\
\text { impedance(ohm) }\end{array}$ & $\begin{array}{c}\text { Loss factor } \\
\text { (V/pC) }\end{array}$ \\
\hline Cavities & & $\sim 14 / 10$ \\
\hline Resistive wall & $2 \mathrm{e}-3$ & 2.0 \\
\hline Masks & $3 \mathrm{e}-2$ & 2.0 \\
\hline Valves & $6 \mathrm{e}-3$ & 0.3 \\
\hline BPMs & $1 \mathrm{e}-4$ & 0.6 \\
\hline IR chambers & $2 \mathrm{e}-3$ & 2.0 \\
\hline Tapers & $2 \mathrm{e}-2$ & 2.0 \\
\hline Bellows & $1 \mathrm{e}-2$ & 2.0 \\
\hline Total & $\sim 0.06$ & $\sim 25 / 21$ \\
\hline
\end{tabular}

The estimated total higher-order-mode power of the electron ring is up to about $240 \mathrm{~kW}$ with $450 \mathrm{~mA}$ current. Compared to B-factories, the HOM power in eRHIC ering is moderate. In case of high intensity operation with 1 A beam current, the HOM energy loss would approach the level of B-factories.

Table 3: HOM power with different loss factor and beam current

\begin{tabular}{|c|c|c|c|}
\hline Loss factor(V/pC) & 15 & 25 & 35 \\
\hline $\mathrm{I}=450 \mathrm{~mA}$ & $120 \mathrm{~kW}$ & $200 \mathrm{~kW}$ & $280 \mathrm{~kW}$ \\
\hline $\mathrm{I}=1000 \mathrm{~mA}$ & $590 \mathrm{~kW}$ & $980 \mathrm{~kW}$ & $1370 \mathrm{~kW}$ \\
\hline
\end{tabular}




\section{Transverse Mode-Coupling Instability (TMCI)}

This instability occurs when two head-tail modes $(\mathrm{m}=0$ and $\mathrm{m}=-1$ in most cases) share the same coherent frequencies. The instability is a severe limitation on the single bunch current in large storage rings with a low beam energy and a low synchrotron tune. Using the estimated transverse wake potential and average beta function of $15 \mathrm{~m}$, it is found that coherent tune shift of the $\mathrm{m}=0$ dipole mode is very small at the design bunch current. The transverse mode-coupling instability threshold is expected to scale as

$$
I_{b}=\frac{4(E / e) \nu_{s}}{\left\langle\operatorname{Im}\left(Z_{\perp}\right) \beta_{\perp}\right\rangle R} \frac{4 \sqrt{\pi}}{3} \sigma_{l}
$$

where $v_{s}$ is the synchrotron tune, $\beta$ is the beta function at the location of the impedance, and $\mathrm{R}$ is the average ring radius. Compared to the $\mathrm{B}$-factory low energy rings, the eRHIC collider has higher energy, higher synchrotron tune, longer bunch length, shorter circumference, and comparable impedance and beta function. The calculated threshold currents are about $46 \mathrm{~mA}$ at $10 \mathrm{GeV}$ and 16 $\mathrm{mA}$, respectively, which are higher than the nominal value $(3.8 \mathrm{~mA})$ with comfortable margins. For all of these reasons the transverse mode-coupling instability threshold will not impose a threat to the performance of the lepton ring.

\section{Longitudinal Microwave Instability Threshold}

The design beta-functions at IP of the lepton beam are about 15 to $30 \mathrm{~cm}$. Since the bunch length of hadron beams is very long (above, say, $10 \mathrm{~cm}$ or longer) the much shorter bunch length of lepton beams $(1 \sim 2 \mathrm{~cm}$ is expected) is not considered to be a problem (hourglass effect is negligible).

\section{Multi-Bunch Instabilities}

Due to the relatively long bunch spacing the multibunch instabilities in this storage ring are not among the major issues. Tables 4 and 5 show the growth times of the fastest growing modes in transverse and longitudinal planes.

Table 4: growth time of transverse modes

\begin{tabular}{|c|c|}
\hline Mode & Growth time (ms) \\
\hline $\mathrm{a}=1$ & $\tau_{1}=228$ \\
& $\tau_{2}=229$ \\
& $\tau_{3}=230$ \\
\hline & $\tau_{1}=2139$ \\
$\mathrm{a}=2$ & $\tau_{2}=2148$ \\
& $\tau_{3}=2153$ \\
\hline
\end{tabular}

The damping times in the electron ring are about $7.4 \mathrm{~ms}$ (transverse) and $3.7 \mathrm{~ms}$ (longitudinal) at $10 \mathrm{GeV}$ and 58 $\mathrm{ms}$ (transverse) and $29 \mathrm{~ms}$ (longitudinal) at $5 \mathrm{GeV}$. The worst situation occurs in the low energy operation, where the coupled-bunch instabilities have the fastest growth rates and damping time is long. The preliminary simulations suggest that a feedback system is needed and sufficient.
Table 5: growth time of longitudinal modes

\begin{tabular}{|c|c|}
\hline Mode & Growth time $(\mathrm{ms})$ \\
\hline \multirow{2}{*}{$=0$} & $\tau_{1}=38$ \\
& $\tau_{2}=55$ \\
& $\tau_{3}=67$ \\
\hline \multirow{2}{a}{$=1$} & $\tau_{1}=164$ \\
& $\tau_{2}=165$ \\
& $\tau_{3}=166$ \\
\hline
\end{tabular}

\section{TWO STREAM INSTABILITIES}

The lepton species in eRHIC required by physics include electrons and positrons. Each operation mode involves two-stream instability, i.e., fast beam-ion effect for electron operations and electron-cloud effect for positron operations, respectively.

\section{Fast Beam-Ion Instabilities (FBII)}

The relatively large bunch spacing in the eRHIC electron ring causes a small ion trapping effect. However, the ions accumulated during a single passage of the bunch can cause a transient instability. This so-called 'fast beamion instability' (FBII) is similar to the multi-bunch beam break-up in a linac. Usually the FBII is more severe in the vertical plane as the vertical emittance is smaller in the lepton machine. According to the linear model [5], the rise time can be described as

$$
\frac{1}{\tau}=\frac{4 d_{g a s} \sigma_{i o n} \beta_{y} N_{b}^{3 / 2} n_{b}^{2} r_{e} r_{p}^{1 / 2} L_{s e p}^{1 / 2} c}{3 \sqrt{3} \sigma_{y}^{3 / 2} \gamma\left(\sigma_{x}+\sigma_{y}\right)^{3 / 2} A^{1 / 2}}
$$

where $d_{\text {gas }}=p / k_{b} T=5.1 \mathrm{E} 13 \mathrm{~m}^{-3}$ is the density of residual gas, $\sigma_{i o n}$ is the ionization cross section, $N_{b}$ is the particle number per bunch, $r_{e}$ and $r_{p}$ are the classical radius of the electron and proton respectively, $L_{s e p}$ is the bunch spacing, $\sigma_{x}$ and $\sigma_{y}$ are the horizontal and vertical beam sizes, and $A$ is the ion mass in unit of proton mass.

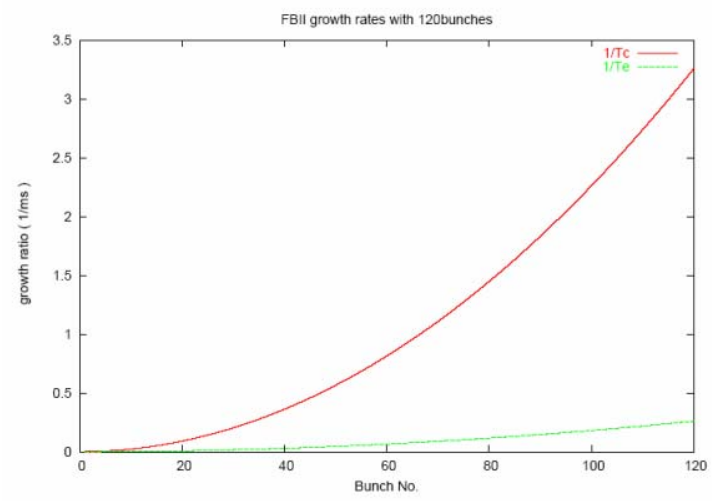

Figure 1: Growth rates of FBII at $5 \mathrm{GeV}$.

Taking into account of the coherent frequency spread, the linear theory gives the coupled bunch motion in the bunch train like $\mathrm{y} \sim \exp \left(\mathrm{t} / \tau_{e}\right)$. The growth time is given by 


$$
\frac{1}{\tau_{e}}=\frac{1}{\tau} \frac{c}{2 \sqrt{2} l_{\text {train }}\left(\Delta \widetilde{\omega}_{i}\right)_{r m s}}
$$

where $\left(\Delta \tilde{\omega}_{i}\right)_{\text {rms }}$ is rms spread of ion coherent angular frequency, $l_{\text {train }}$ is bunch train length.

$$
\omega_{i}=\left(\frac{4 N_{b} r_{p} c^{2}}{3 A L_{s e p} \sigma_{y}\left(\sigma_{x}+\sigma_{y}\right)}\right)^{1 / 2}
$$

The growth rates of FBII at $5 \mathrm{GeV}$ are shown in Figure 1.

\section{Electron Cloud Effects (ECE)}

In the positron-hadron collision operation of eRHIC, the photo electrons generated by synchrotron radiation hitting the vacuum chamber walls, and secondary emission due to multipacting in the presence of the electric field of the positron beam, can accumulate in the beam pipe during the multi-bunch operation with short bunch spacing. This gives rise to a so-called 'electron cloud' (EC). Several effects have been observed in different machines, including

- Pressure rise

- Beam-size blow-up

- Coupled-bunch instability, etc. Multi-bunch effect:

For coupled-bunch instability due to EC, if we assume that the density of the electron cloud is saturated, then the growth time can be estimated as [10]

$$
\tau_{C B}=\frac{\gamma \omega_{\beta} h_{x} h_{y} L_{s e p}}{2 r_{e} N_{b} c^{2}}
$$

$\gamma$ is relative energy factor, $\omega_{\beta}$ is betatron frequency, $\mathrm{h}_{\mathrm{x}}$, $\mathrm{h}_{\mathrm{y}}$ are transverse dimensions of the vacuum chamber, $L_{\text {sep }}$ is bunch spacing, $\mathrm{N}_{\mathrm{b}}$ is number of particles per bunch. Assuming similar vacuum chamber dimensions to that of the existing lepton machines, the growth time is at the level of $1.0 \mathrm{~ms}$ in e+ operation.

Single bunch effect:

The electron cloud can also drive single bunch instability. Here we use treat it as a transverse modecoupling instability. With a two-particle model, the threshold electron density of TMCI is [11]

$$
\rho_{e, \text { threshold }} \approx \frac{2 \gamma_{s}}{\pi \beta_{y} r_{e} C}
$$

$\mathrm{C}$ is ring circumference, $v_{s}$ is synchrotron tune. The threshold is about $1.2 \times 10^{13}$ at $10 \mathrm{GeV}$ and $0.6 \times 10^{13}$ at 5 $\mathrm{GeV}$, respectively. The preliminary simulation shows that the electron cloud density in eRHIC lepton ring could approach this level if no precautionary measure is taken. It is worth nothing that above models may overestimate the effects in some extent.

The major cures include:

- a vacuum ante-chamber

- coating of the chamber with TiN or NEG

- installation of solenoid coils

The first two measures may reduce the electron cloud density by a factor $3 \sim 10$. The solenoids field (20 30
Gauss) also prove to be a very effective method to suppress the electron cloud effects in low energy rings of B-Factories[7][8]. The eRHIC lepton ring will adopt the ante-chamber concept with proper coatings in vacuum system designs and the solenoid coils can be the backup solution. The detailed simulation studies are underway.

\section{SUMMARY}

The preliminary investigations of the major expected instabilities in the lepton ring of eRHIC have been made through analytical calculations, simulations, and scaled performance from the other lepton machines, mainly Bfactories. While conventional impedance-related beam instabilities do not threat on operation of the lepton ring of eRHIC the two-stream instabilities, mainly FBII and ECE, are major concerns. The current studies found that these two effects are not more serious than those in the existing B-factories. These estimates indicate that good engineering design and feedback can limit the instabilities to a similar or lower level than the B-factories at similar energy.

\section{ACKNOWLEDGMENTS}

We'd like to thanks R. Milner, T. Roser for their support in the studies. We also want to thank M. Billing, W. Franklin, H. Fukuma, H. Hseuh, K. Ohmi, U. Wienands, F. Zimmermann for helpful discussions.

\section{REFERENCES}

[1] T. Ieiri, et al., Impedance Measurements in the KEKB, EPAC 2000, Vienna (2000)

[2] R. Rimmer, J. Bird, D. Li, Comparison of calculated, measured, and beam sampled impedances of a higherorder-mode-damped rf cavity, Physical Review Special Topics, Volume 3, 102001(2000)

[3] PEP-II, an Asymmetric B Factory: A Conceptual Design Report, SLAC-R-418 (1993)

[4] KEKB B-factory Design Report, KEK Report 95-7 (1995)

[5] T. Raubenheimer and F. Zimmermann, Physical Review E 52, no.5, p.5487 (1995)

[6] M. Zisman, S. Chattopadhyay, J. Bisognano, ZAP User's Manual, LBL-21270 (1986)

[7] J. Seeman, Invited Talk in Super B-Factory Workshop in Hawaii, Honolulu, Jan. 19 22, 2004 (2004)

[8] J. Flanagan, Invited talk in Super B-Factory Workshop in Hawaii, Honolulu, Jan. 19 22, 2004(2004)

[9] A. Chao, M. Tigner, Handbook of Accelerator Physics and Engineering, World Scientific(1999)

[10] F. Zimmermann, G, Rumolo, APAC 2001, 352 (2001)

[11] K. Ohmi, F. Zimmermann, Physical Review Letters $85,3821(2000)$

[12] D. H. Rubin, et al., CESR Status and Performance, PAC 2001, Chicago, 3520(2001)

[13] M. Farkhondeh, V. Ptitsyn, (edit), eRHIC ZerothOrder Design Report (2004) 